\title{
Total synthesis of $(+)$-azimine via diastereoselective aminopalladation
}

Yuji Kurogome, ${ }^{\mathrm{a}}$ Masaya Kogiso, ${ }^{\mathrm{a}}$ Kok Kong Looi, ${ }^{\mathrm{b}}$ Yasunao Hattori, ${ }^{\mathrm{c}}$ Hiroyuki Konno, ${ }^{\mathrm{d}}$ Mitsuru Hirota, ${ }^{\mathrm{b}}$ and Hidefumi Makabe ${ }^{a *}$

${ }^{\mathrm{a}}$ Graduate School of Agriculture, Sciences of Functional Foods, Shinshu University,

8304 Minami-minowa, Kami-ina, Nagano, 399-4598, Japan

${ }^{\mathrm{b}}$ Department of Bioscience and Biotechnology, Faculty of Agriculture, Shinshu University, 8304 Minami-minowa, Kami-ina, Nagano, 399-4598, Japan

${ }^{\mathrm{c}}$ Department of Medicinal Chemistry, Kyoto Pharmaceutical University, Yamashina-ku, Kyoto 607-8412, Japan

${ }^{\mathrm{d} D e p a r t m e n t ~ o f ~ B i o c h e m i c a l ~ E n g i n e e r i n g, ~ G r a d u a t e ~ S c h o o l ~ o f ~ S c i e n c e ~ a n d ~ T e c h n o l o g y, ~ Y a m a g a t a ~ U n i v e r s i t y, ~}$ Yonezawa, Yamagata 992-8510, Japan

*Corresponding author. Tel. +81 26577 1630; fax +81 26577 1700; e-mail: makabeh@shinshu-u.ac.jp

Abstract: The aminopalladation of amino allylic alcohol using $\mathrm{Cl}_{2} \mathrm{Pd}(\mathrm{MeCN})_{2}$ in $\mathrm{CH}_{2} \mathrm{Cl}_{2}$ gave the 2,6-disubstituted piperidine with excellent diastereoselectivity. This compound was successfully converted into (+)-azimine (1) using cross-metathesis and Shiina macrolactonization.

Key words: alkaloids, piperidine, natural product, aminopalladation

\section{Introduction}

Among a lot of numbers of biologically active natural compounds, the alkaloids are most paid attention due to their significant biological activities and unique structures. ${ }^{1,2}$ Most of piperidine alkaloids possess a chiral center at C2 and/or C6 position, thus stereoselective construction is very important. For example, excellent diastereoselective syntheses have been achieved as follows. Stereoselective synthesis of trans-2,6-disubstituted piperidine alkaloids using $\operatorname{Pd}(0)$ catalyzed $N$-alkylation has been achieved by Tadano in $1993 .{ }^{3}$ In 2000, Hirai reported $\mathrm{Pd}(\mathrm{II})$ catalyzed cyclization of amino allylic alcohol to afford 2-substituted piperidine with excellent diastereoselectivity. ${ }^{4}$

While most of alkaloids generally exist as monomers, (+)-azimine (1) is macrocyclic dilactone, which was isolated from Azima tetracantha L. ${ }^{5,6}$ Structually, azimine (1) is a dimer of (+)-azimic acid (2) which has 2-methyl-3-piperidinol skeleton with a carboxyl group at terminal position. This compound is presumed 
biosynthetic and synthetic precursor of (+)-azimine (1) (Figure 1). The syntheses of (+)-azimic acid (2) were reported by many researchers, ${ }^{7}$ however, there are only one example of asymmetric total synthesis of $(+)$-azimine (1) by Kibayashi and co-workers using stereoselective intramolecular hetero-Diels-Alder reaction of an acylnitroso compound. ${ }^{8}$
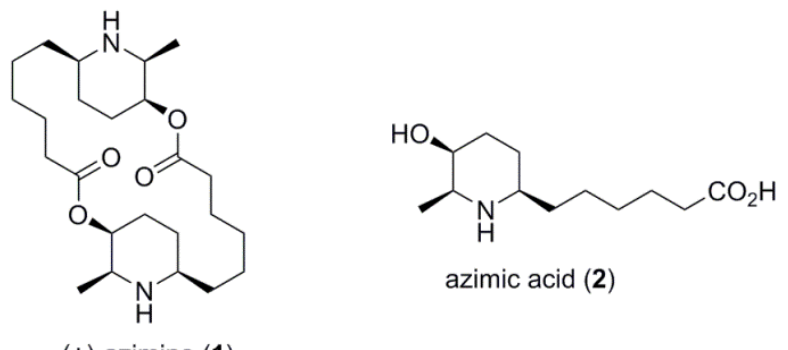

Figure 1. The structures of (+)-azimine (1) and (+)-azimic acid (2).

In our previous report, we accomplished an asymmetric total synthesis of (-)-cassine using diastereoselective aminopalladation, however, the yield of this reaction was not high enough. ${ }^{9}$ Therefore, we have investigated to improve the yield and found that the effect of solvent was useful for diastereoselective aminopalladation. Here we wish to report improved diastereoselective Pd(II)-catalyzed cyclization and its application to the total synthesis of azimine (1).

\section{Results and discussion}

Scheme 1 outlines our synthetic strategy. (+)-Azimine (1) would be derived from $\mathbf{3}$ via macrolactonization. Hydroxy caboxylic acid 3 would be prepared via several steps from 4a and methyl 5-hexenate including Grubbs cross-metathesis. Piperidine $\mathbf{4 a}$ would be synthesized using similar procedure from $\mathbf{5}$ as we reported previously (Scheme 1). ${ }^{9}$

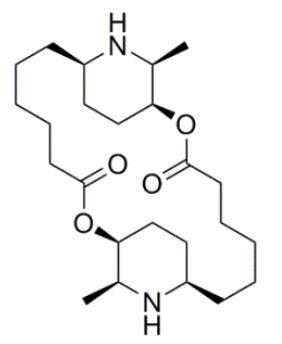

(+)-azimine (1)

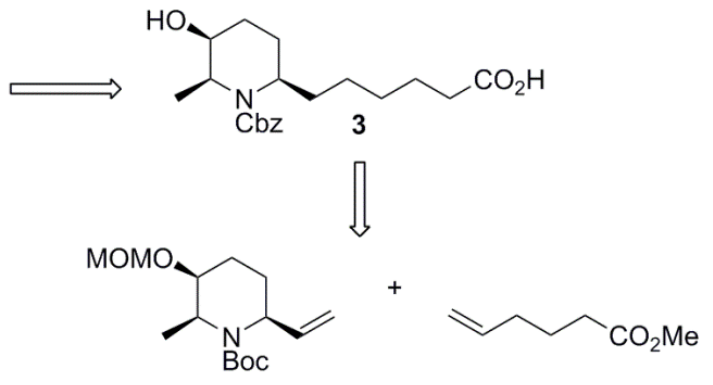

$4 a$

If
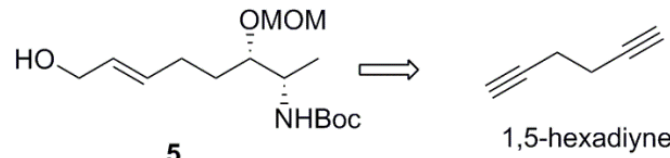

Scheme 1. Synthetic strategy of (+)-azimine (1). 
The cyclization precursor 5 was synthesized from 1,5-hexadiyne as we reported earlier. ${ }^{9}$ The results of diastereoselective aminopalladation of $\mathbf{5}$ and its derivatives are summarized in Table 1. At first we used the reaction condition as we have reported before to give $\mathbf{4 a}$ in a moderate yield. ${ }^{9}$ Using $\mathrm{Pd}(\mathrm{II})$ catalysts with phosphine ligands such as $\mathrm{PPh}_{3}$ and dppf did not afford cyclized product. We also examined allylic ester such as pivaloyl, mesityl, and biphenyl esters because Hirai reported Pd(II) catalyzed cyclization of amino allylic pivaloyl ester afforded 3,4,5,6-substituted piperidine with excellent diastereoselectivity in $36 \%$ yield. ${ }^{4}$ However, the yield of cyclized product was very low in our cases. We found that using allylic ester was difficult to construct 2,6-piperidine ring. Finally, we switched the solvent from THF to $\mathrm{CH}_{2} \mathrm{Cl}_{2}$ to afford $4 \mathbf{a}$ in good yield.

Table 1. Stereoselective aminopalladation of $\mathbf{5}$ and its derivatives.

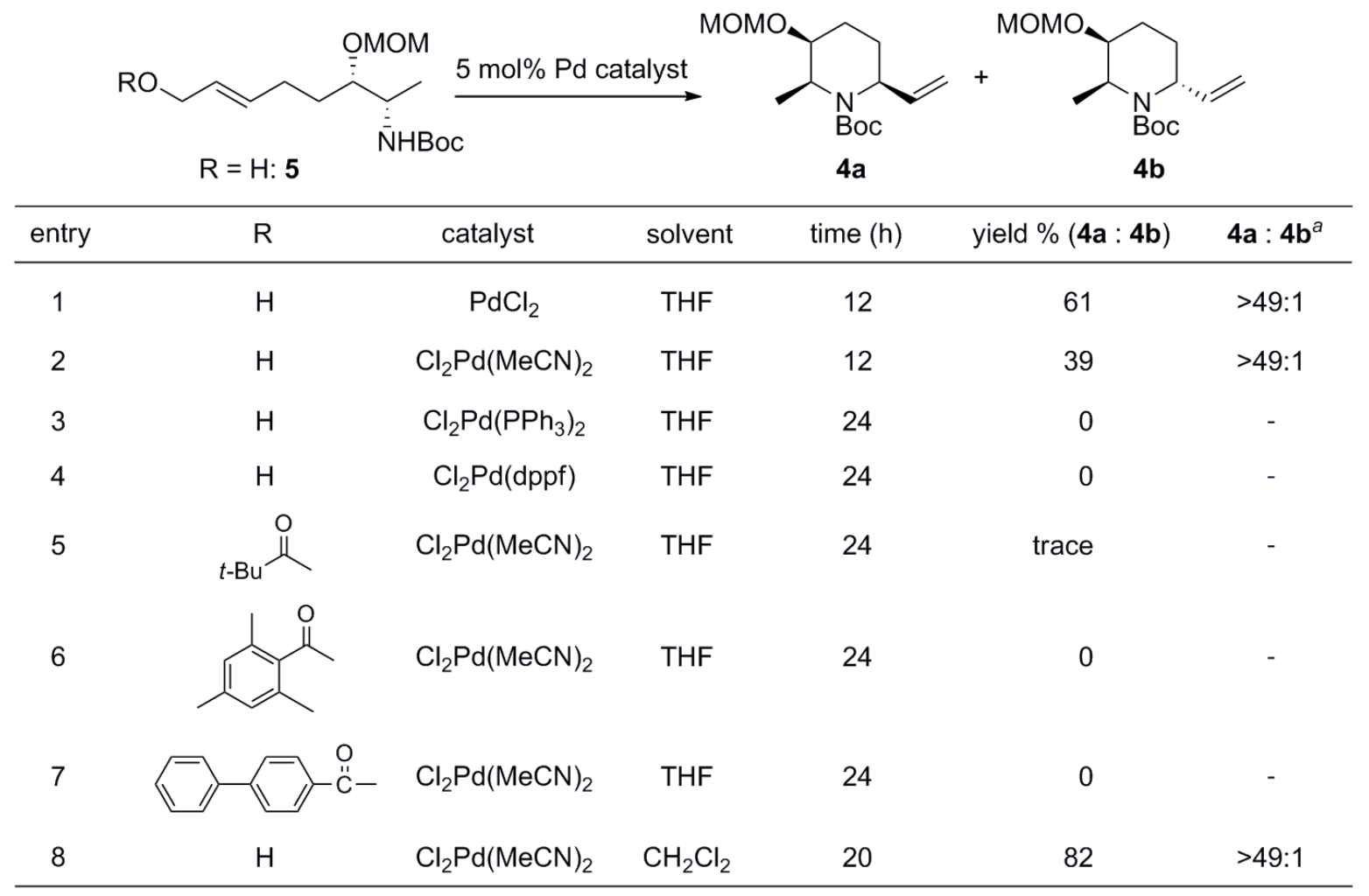

${ }^{a}$ The relative stereochemistry of $4 a$ was determined by $2 \mathrm{D}-\mathrm{NOESY}$ experiment. ${ }^{9}$

Because we have optimized the reaction condition for stereoselective aminopalladation to prepare $\mathbf{4 a}$, we began total synthesis of (+)-azimine (1). Cross-metathesis between 4a and methyl 5-hexenate using second generation Hoveyda-Grubbs catalyst afforded chain elongated compound 6 in $88 \%$ yield. ${ }^{10}$ Using second generation of Grubbs catalyst gave 6 only in 14\% yield. The compound 6 was subjected to catalytic hydrogenation using 5\% Pearlman's catalyst to give saturated product 7. Deprotection of the MOM and the Boc groups with $\mathrm{HCl}$ in $\mathrm{MeOH}$ subsequent treatment with $\mathrm{CbzCl}$ and $\mathrm{NaHCO}_{3}$ afforded $\mathbf{8}$. ${ }^{11}$ Treatment of $\mathbf{8}$ with $\mathrm{Ba}(\mathrm{OH})_{2} \cdot 8 \mathrm{H}_{2} \mathrm{O}$ afforded hydroxy carboxylic acid 9 whose ${ }^{1} \mathrm{H}$ NMR, ${ }^{13} \mathrm{C}$ NMR data were in good agreement with those of the reported values by Kibayashi and co-workers. ${ }^{8}$ Macrocyclic dilactonization of 9 was attempted using Yamaguchi 
macrolactonization as reported Kibayashi and co-workers. ${ }^{8}$ Although Kibayashi et al. reported this reaction was proceeded in $71 \%$ yield, actually the yield from 9 to $\mathbf{1 0}$ was only $34 \%$ in our experiment. Thus we switched to use Shiina macrolactonization to generate 10 in $68 \%$ yield. ${ }^{12}$ Yamaguchi's method was generally carried out using large excess amount of base. ${ }^{13}$ Longer reaction time might cause decomposition of macrolactone 9. On the other hand, Shiina's protocol did not need to use excess amount of base. Finally, hydrogenolysis of the Cbz group furnished (+)-azimine (1). The optical rotation of synthetic 1 was consistent with $\left\{[\alpha]^{20}{ }_{D}+3.10(c=0.30, \mathrm{EtOH})\right\}$ that reported by Kibayashi and co-workers $\left.\{[\alpha]]^{25}+3.14(c=0.74, \mathrm{EtOH})\right\} .{ }^{8}$ The ${ }^{1} \mathrm{H}$ NMR, ${ }^{13} \mathrm{C}$ NMR, and MS spectra and melting point of synthetic $\mathbf{1}$ were also in good agreement with those of the reported values (Scheme $2) .^{8}$

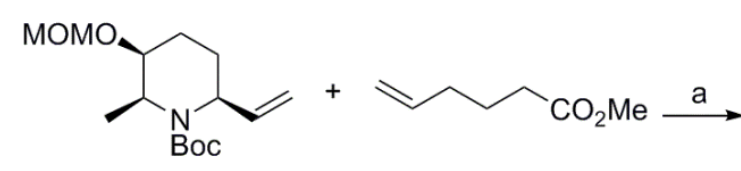

$4 a$

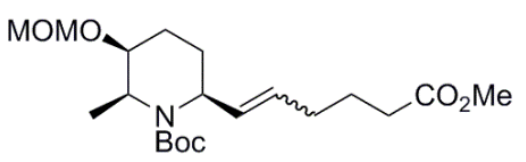

$6(E: Z=9: 1)$

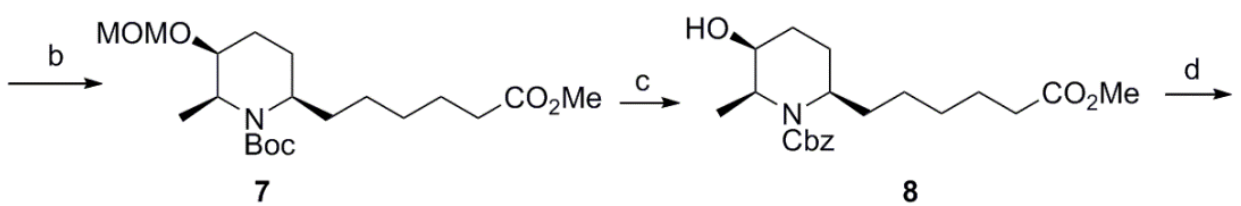

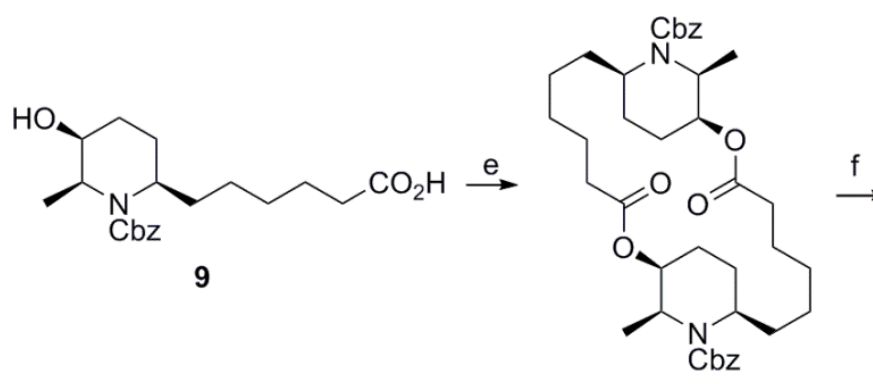

10

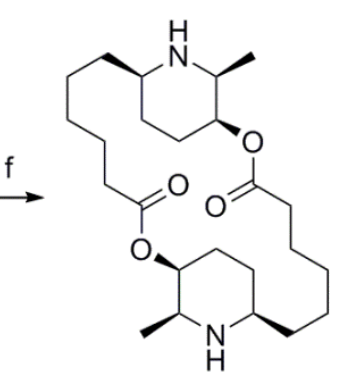

$(+)$-azimine (1)

Scheme 2. Synthesis of (+)-azimine (1).

Regents and conditions: (a) Hoveyda-Grubbs 2nd catalyst, $\mathrm{CH}_{2} \mathrm{Cl}_{2}$, reflux, 88\%; (b) $\mathrm{H}_{2}, 5 \% \mathrm{Pd}(\mathrm{OH})_{2} / \mathrm{C}$, 99\%; (c) (i) $\mathrm{HCl}, \mathrm{MeOH}$, (ii) $\mathrm{CbzCl}, \mathrm{NaHCO}_{3}$, dioxane- $\mathrm{H}_{2} \mathrm{O}, 73 \%$; (d) $\mathrm{Ba}(\mathrm{OH})_{2} \cdot 8 \mathrm{H}_{2} \mathrm{O}, \mathrm{MeOH}, 40{ }^{\circ} \mathrm{C}, 85 \%$; (e) Shiina macrolactonization, $68 \%$; (f) $\mathrm{H}_{2}, 5 \% \mathrm{Pd}(\mathrm{OH})_{2} / \mathrm{C}$, quant.

\section{Conclusion}

In conclusion, we have achieved a total synthesis of (+)-azimine (1) using a diastereoselective aminopalladation. The key intermediate $4 \mathbf{a}$ can be used as building blocks for synthesizing other cis-2,6-disubstituted piperidine alkaloids.

\section{Experimental}


4.1. General. All melting points were uncorrected. ${ }^{1} \mathrm{H}$ and ${ }^{13} \mathrm{C}$ NMR spectra were measured with a Bruker DRX 500 FT-NMR spectrometer in $\mathrm{CDCl}_{3}$ at 500 and $125 \mathrm{MHz}$, respectively. Chemical shifts were relative to tetramethylsilane as an internal standard. The coupling constants were given in Hz. Mass spectra were obtained on JEOL JMS-700, JEOL JMS-SX102A and TMS-T100 LC mass spectrometer. IR spectra were recorded with JASCO FT-IR 480 Plus infrared spectrometer. Optical rotations were determined with a JASCO DIP-1000 polarimeter.

4.1.1. $(2 S, 3 S, 6 S)$-2-Methyl- $N$-tert-butoxycarbonyl-3-methoxymethoxy-6-vinylpiperidine (4a). To a solution of 5 (116 mg, $0.38 \mathrm{mmol})$ in $\mathrm{CH}_{2} \mathrm{Cl}_{2}(5 \mathrm{~mL})$ was added $\mathrm{Cl}_{2} \mathrm{Pd}\left(\mathrm{CH}_{3} \mathrm{CN}\right)_{2}(5 \mathrm{mg}, 19 \mu \mathrm{mol})$ at $0{ }^{\circ} \mathrm{C}$ and the mixture was stirred for $12 \mathrm{~h}$ at room temperature. The reaction mixture was diluted with water and extracted with EtOAc.

The organic layer was washed with brine, dried over $\mathrm{MgSO}_{4}$ and concentrated. The residue was purified by preparative TLC (hexane : AcOEt $\left.=5: 1, \mathrm{R}_{\mathrm{f}}=0.36\right)$ to give $\mathbf{4 a}(89 \mathrm{mg}, 82 \%)$ as a colorless oil. $[\alpha]^{19}-47(c 1.1$, $\mathrm{CHCl}_{3}$ ). IR (film) $v_{\max } \mathrm{cm}^{-1}:$ 3080, 2980, 2930, 2880, 1690, 1450, 1395, 1370, 1240, 1170, 1100, 1040, $965,920$. ${ }^{1} \mathrm{H}$ NMR $\delta: 1.12(3 \mathrm{H}, \mathrm{d}, J=7.0 \mathrm{~Hz}), 1.47(9 \mathrm{H}, \mathrm{s}), 1.60-1.75(3 \mathrm{H}, \mathrm{m}), 1.96(1 \mathrm{H}, \mathrm{m}), 3.37(3 \mathrm{H}, \mathrm{s}), 3.66(1 \mathrm{H}, \mathrm{m})$, $4.46(1 \mathrm{H}, \mathrm{qd}, J=6.6,6.6 \mathrm{~Hz}), 4.63(1 \mathrm{H}, \mathrm{brs}), 4.67(2 \mathrm{H}, \mathrm{s}), 5.08(1 \mathrm{H}, \mathrm{d}, J=10.6 \mathrm{~Hz}), 5.13(1 \mathrm{H}, \mathrm{d}, J=17.4 \mathrm{~Hz})$, $5.91(1 \mathrm{H}, \mathrm{ddd}, J=17.0,10.5,5.3 \mathrm{~Hz}) .{ }^{13} \mathrm{C} \mathrm{NMR} \delta: 14.1,21.0,26.3,28.4,49.5,50.7,55.4,74.7,79.7,95.0,114.6$, 140.0, 155.1. HREIMS [M] $]^{+}$Found, 285.1947, Calcd. for $\mathrm{C}_{15} \mathrm{H}_{27} \mathrm{NO}_{4}: 285.1940$.

\subsubsection{Methyl 6-\{(2S,3S,5EZ,6S)-1-[(tert-butoxy)carbonyl]-5-methoxymethoxy-6-methylpiperidine-2-yl\}-} hex-5-enoate (6). To a solution of $4 \mathbf{a}(29 \mathrm{mg}, 0.10 \mathrm{mmol})$ and methyl hex-5-enoate $(33 \mathrm{mg}, 0.26 \mathrm{mmol})$ in $\mathrm{CH}_{2} \mathrm{Cl}_{2}(5.0 \mathrm{~mL})$ was added Hoveyda-Grubbs $2^{\text {nd }}$ catalyst $(6.4 \mathrm{mg}, 0.010 \mathrm{mmol})$. After being stirred for $3 \mathrm{~h}$ under reflux, additional solution of Hoveyda-Grubbs $2^{\text {nd }}$ catalyst in $\mathrm{CH}_{2} \mathrm{Cl}_{2}, 6.4 \mathrm{mg}, 0.010 \mathrm{mmol}$ ) and methyl 5-hexenoate ( $33 \mathrm{mg}, 0.26 \mathrm{mmol}$ ) was added. After being stirred for $3 \mathrm{~h}$ under reflux, the solvent was removed and the residue was purified by preparative TLC (hexane : AcOEt $\left.=10: 1, \mathrm{R}_{\mathrm{f}}=0.24\right)$ to give $6(35 \mathrm{mg}, 88 \%, E: Z=$ 9:1) as a colorless oil. $[\alpha]^{20}{ }_{D}-21\left(c 0.25, \mathrm{CHCl}_{3}\right)$. IR (film) $v_{\max } \mathrm{cm}^{-1}: 2945,2848,1739,1688,1366,1246,1173$, 1043, 965. ${ }^{1} \mathrm{H}$ NMR ( $E$ isomer) $\delta: 1.12(3 \mathrm{H}, \mathrm{d}, J=7.0 \mathrm{~Hz}), 1.46(9 \mathrm{H}, \mathrm{s}), 1.62-1.87(6 \mathrm{H}, \mathrm{m}), 2.05(2 \mathrm{H}, \mathrm{m}), 2.32$ $(2 \mathrm{H}, \mathrm{t}, J=7.5 \mathrm{~Hz}), 3.38(3 \mathrm{H}, \mathrm{s}), 3.64(1 \mathrm{H}, \mathrm{m}), 3.66(3 \mathrm{H}, \mathrm{s}), 4.44(1 \mathrm{H}, \mathrm{m}), 4.60(1 \mathrm{H}, \mathrm{brs}), 4.67(2 \mathrm{H}, \mathrm{s}), 5.53(2 \mathrm{H}$, m). ${ }^{13} \mathrm{C}$ NMR ( $E$ isomer) $\delta: 14.4,21.1,24.4,27.1,28.5,31.8,33.3,49.3,51.4,55.4,74.7,79.6,95.0,129.7,132.5$, 155.1, 174.0. HRFABMS [M+H]+: Found, 386.2549, Calcd. for $\mathrm{C}_{20} \mathrm{H}_{36} \mathrm{NO}_{6}: 386.2543$.

\subsection{3.}

Methyl

6-\{(2S,3S,6R)-1-[(tert-butoxy)carbonyl]-5-methoxymethoxy-6-methylpiperidine-2-yl\}-hexanoate (7). The mixture of $6(49 \mathrm{mg}, 0.13 \mathrm{mmol})$ and $\mathrm{Pd}(\mathrm{OH})_{2} / \mathrm{C}(5 \% \mathrm{w} / \mathrm{w}, 2.5 \mathrm{mg})$ in $\mathrm{MeOH}(4.0 \mathrm{~mL})$ was stirred under hydrogen atmosphere. After being stirred for $5 \mathrm{~h}$, the reaction mixture was filtered and concentrated. The residue 
was purified by preparative TLC (hexane : AcOEt $\left.=10: 1, \mathrm{R}_{\mathrm{f}}=0.25\right)$ to afford $7(49 \mathrm{mg}, 99 \%)$ as a colorless oil. $[\alpha]^{20}{ }_{\mathrm{D}}-12.8\left(c 0.18, \mathrm{CHCl}_{3}\right)$. IR (film) $v_{\max } \mathrm{cm}^{-1}: 2944,2861,1740,1686,1439,1405,1366,1321,1174,1146$, 1043, 918. ${ }^{1} \mathrm{H}$ NMR $\delta: 1.13$ (3H, d, $\left.J=7.0 \mathrm{~Hz}\right), 1.25-1.31(12 \mathrm{H}, \mathrm{m}), 1.46(9 \mathrm{H}, \mathrm{s}), 2.32(2 \mathrm{H}, \mathrm{m}), 3.38(3 \mathrm{H}, \mathrm{s}), 3.63$ $(1 \mathrm{H}, \mathrm{m}), 3.67(3 \mathrm{H}, \mathrm{s}), 3.99$ (1H, brs), $4.43(1 \mathrm{H}, \mathrm{brs}), 4.67(2 \mathrm{H}, \mathrm{s}) .{ }^{13} \mathrm{C}$ NMR $\delta: 14.3,20.7,24.9,26.4,27.5,28.5$, 29.1, 34.0, 34.8, 49.7, 51.4, 55.4, 74.9, 79.4, 94.9, 155.2, 174.1. HRFABMS [M+H] ${ }^{+}$: Found, 388.2693, Calcd. for $\mathrm{C}_{20} \mathrm{H}_{38} \mathrm{NO}_{6}: 388.2699$.

\subsubsection{Methyl 6-\{(2S,3S,6R)-1-[(Benzyloxy)carbonyl]-5-hydroxy-6-methylpiperidine-2-yl $\}$-hexanoate (8). To} a solution of 7 (47 mg, $0.12 \mathrm{mmol})$ in $\mathrm{MeOH}(2.0 \mathrm{~mL})$ was added a few drops of conc. $\mathrm{HCl}$. After being stirred for $19 \mathrm{~h}$, the mixture was extracted with $\mathrm{CHCl}_{3}$, washed with $10 \%$ aqueous $\mathrm{NH}_{3}$, brine, dried over $\mathrm{K}_{2} \mathrm{CO}_{3}$. Concentration afforded crude hydroxyamine which was used for the next step without further purification. To a solution of hydroxyl amine in 1,4-dioxane $(1.0 \mathrm{~mL})$ and $\mathrm{H}_{2} \mathrm{O}(1.0 \mathrm{~mL})$ was added $\mathrm{CbzCl}(0.18 \mathrm{~mL}, 1.23 \mathrm{mmol})$. After being stirred for $72 \mathrm{~h}$, the reaction was quenched with water and the mixture was extracted with EtOAc, washed with saturated aqueous $\mathrm{NaHCO}_{3}$, brine, and dried over $\mathrm{MgSO}_{4}$. Concentration and purification by preparative TLC (hexane : AcOEt $\left.=2: 1, \mathrm{R}_{\mathrm{f}}=0.22\right)$ afforded $\mathbf{8}\left(46 \mathrm{mg}, 73 \%, 2\right.$ steps) as a colorless oil. $[\alpha]^{19} \mathrm{D}$ -4.7 (c 0.46, $\mathrm{CHCl}_{3}$ ). IR (film) $v_{\max } \mathrm{cm}^{-1}: 3448,2942,2861,1737,1692,1497,1412,1299,1172,1071,1029,737$, 699, 669. ${ }^{1} \mathrm{H}$ NMR $\left(\mathrm{CDCl}_{3}\right) \delta: 1.13(3 \mathrm{H}, \mathrm{d}, J=7.0 \mathrm{~Hz}), 1.22-1.75(13 \mathrm{H}, \mathrm{m}), 2.27(2 \mathrm{H}, \mathrm{t}, J=7.5 \mathrm{~Hz}), 3.67(3 \mathrm{H}, \mathrm{s})$, $3.76(1 \mathrm{H}, \mathrm{m}), 4.10(1 \mathrm{H}, \mathrm{brs}), 4.50(1 \mathrm{H}, \mathrm{m}), 5.16(2 \mathrm{H}, \mathrm{m}), 7.29-7.40(5 \mathrm{H}, \mathrm{m}) .{ }^{13} \mathrm{C}$ NMR $\delta: 13.7,23.0,24.8,26.4$, 26.9, 29.0, 34.0, 34.7, 49.9, 50.8, 51.4, 67.1, 69.8, 127.9, 128.5, 136.9, 155.9, 174.1. HRFABMS [M+H]+: Found, 378.2284, Calcd. for $\mathrm{C}_{21} \mathrm{H}_{32} \mathrm{NO}_{5}: 378.2280$.

4.1.5. 6-\{(2R,5S,6S)-1-[(Benxyloxy)carbonyl]-5-hydroxy-6-methylpiperidine-2-yl\}hexanoic acid (9). To a solution of $8(46 \mathrm{mg}, 0.12 \mathrm{mmol})$ in $\mathrm{MeOH}(2.8 \mathrm{~mL})$ was added $\mathrm{Ba}(\mathrm{OH})_{2} \cdot 8 \mathrm{H}_{2} \mathrm{O}(194 \mathrm{mg}, 0.60 \mathrm{mmol})$. After being stirred for $48 \mathrm{~h}$ at $40{ }^{\circ} \mathrm{C}$, the reaction was quenched with $1 \mathrm{~N} \mathrm{HCl}$ and the mixture was extracted with diethyl ether, washed with water, brine, and dried over $\mathrm{MgSO}_{4}$. The residue was purified by preparative TLC $\left(\mathrm{CHCl}_{3}: \mathrm{MeOH}=5: 1, \mathrm{R}_{\mathrm{f}}=0.21\right)$ to afford $9(37 \mathrm{mg}, 85 \%)$ as a colorless oil. $[\alpha]^{20}{ }_{\mathrm{D}}-8.42\left(c 1.04, \mathrm{CHCl}_{3}\right) . \mathrm{IR}$ (film) $v_{\max } \mathrm{cm}^{-1}: 3419,2939,2860,1669,1540,1497,1417,1300,1145,1096,1069,1027,770,736,698,668 .{ }^{1} \mathrm{H}$ $\operatorname{NMR}\left(\mathrm{CDCl}_{3}\right) \delta: 1.15(3 \mathrm{H}, \mathrm{d}, J=7.0 \mathrm{~Hz}), 1.22-1.75(12 \mathrm{H}, \mathrm{m}), 2.24(2 \mathrm{H}, \mathrm{t}, J=7.5 \mathrm{~Hz}), 3.74(1 \mathrm{H}, \mathrm{m}), 4.11(1 \mathrm{H}$, brs), 4.48 (1H, m), 5.14 (2H, m), 6.10-6.70 (2H, br.), 7.29-7.41 (5H, m). ${ }^{13} \mathrm{C}$ NMR $\delta: 13.7,22.9,24.8,26.4,26.8$, 26.9, 29.0, 34.3, 34.5, 49.9, 50.9, 67.2, 69.7, 127.8, 128.0, 128.5, 136.7, 156.0, 179.3. HRFABMS [M+H] Found, 364.2128, Calcd. for $\mathrm{C}_{20} \mathrm{H}_{30} \mathrm{NO}_{5}$ : 364.2124 .

4.1.6.

Dibenzyl

$(1 S, 9 R, 11 S, 12 S, 20 R, 22 S)-11,22$-dimethyl-3,14-dioxa-2,13-dioxo-10,21-diazatricyclo[18.2.2.2 $\left.{ }^{9,12}\right]$ hexacosane10,21-dicarboxylate (10). To a solution of 2-methyl-6-nitrobenzoic anhydride (43 mg, $0.13 \mathrm{mmol}$ ) and DMAP 
(49 mg, $0.4 \mathrm{mmol})$ in $\mathrm{CH}_{2} \mathrm{Cl}_{2}(21 \mathrm{~mL})$ was added a solution of 9 (37 mg, $\left.0.10 \mathrm{mmol}\right)$ in THF (34 mL) over $6 \mathrm{~h}$. After being stirred for $1 \mathrm{~h}$, the reaction was quenched with sat. $\mathrm{NaHCO}_{3}$ aq. at $0^{\circ} \mathrm{C}$ and the mixture was extracted with diethyl ether, washed with water, brine, and dried over $\mathrm{Na}_{2} \mathrm{SO}_{4}$. Concentration and purification by preparative TLC (hexane : EtOAc : ether $\left.=2: 2: 1, \mathrm{R}_{\mathrm{f}}=0.16\right)$ afforded $\mathbf{1 0}(24 \mathrm{mg}, 68 \%)$ as a colorless oil. $[\alpha]^{20}{ }_{\mathrm{D}}$ -14 (c 0.40, $\mathrm{CHCl}_{3}$ ). IR (film) $v_{\max } \mathrm{cm}^{-1}: 2942,2860,1733,1694,1497,1455,1411,1381,1298,1236,1150$, 1120, 1087, 1022, 753, 698; ${ }^{1} \mathrm{H}$ NMR $\left(\mathrm{CDCl}_{3}, 1.35: 1\right.$ amide rotamers) $\delta: 1.21(2.6 \mathrm{H}, \mathrm{d}, J=7.0 \mathrm{~Hz}), 1.25(3.4 \mathrm{H}$, $\mathrm{d}, J=7.0 \mathrm{~Hz}), 1.20-1.85(24 \mathrm{H}, \mathrm{m}), 2.21-2.43(4 \mathrm{H}, \mathrm{m}), 4.15(0.85 \mathrm{H}, \mathrm{brs}), 4.20(1.15 \mathrm{H}, \mathrm{brs}), 4.56(0.85 \mathrm{H}, \mathrm{q}, J=$ $7.0 \mathrm{~Hz}), 4.64(1.15 \mathrm{H}, \mathrm{q}, J=7.0 \mathrm{~Hz}), 4.84-4.92(2 \mathrm{H}, \mathrm{m}), 5.13(4 \mathrm{H}, \mathrm{brs}), 7.29-7.38(10 \mathrm{H}, \mathrm{m}) .{ }^{13} \mathrm{C}$ NMR $\delta: 16.1$, 16.20, 20.1, 20.2, 24.4, 24.5, 25.3, 25.6, 25.8, 26.0, 28.5, 28.7, 29.7, 33.7, 33.9, 34.4, 34.7, 47.9, 48.0, 49.8, 49.9, 67.2, 67.3, 70.7, 70.8, 128.0, 128.5, 136.7, 155.7, 172.1, 172.2. HRFABMS [M+H] ${ }^{+}$: Found, 691.3955, Calcd. for $\mathrm{C}_{40} \mathrm{H}_{55} \mathrm{~N}_{2} \mathrm{O}_{8}: 691.3958$.

4.1.7. (+)-Azimine (1). The mixture of $10(7.0 \mathrm{mg}, 0.01 \mathrm{mmol})$ and $\mathrm{Pd}(\mathrm{OH})_{2} / \mathrm{C}(5 \% \mathrm{w} / \mathrm{w}, 0.4 \mathrm{mg})$ in $\mathrm{MeOH}(0.4$ $\mathrm{mL}$ ) was stirred under hydrogen atmosphere. After being stirred for $20 \mathrm{~h}$, the reaction mixture was filtered and concentrated to afford 1 (6.2 mg, quant.) as a colorless solid. Mp $111-112^{\circ} \mathrm{C},[\alpha]^{20} \mathrm{D}+3.10(c 0.30$, EtOH). IR (film) $v_{\max } \mathrm{cm}^{-1}: 3390,2930,2856,1728,1252,1157,1114,1075,973 ;{ }^{1} \mathrm{H}$ NMR $\left(\mathrm{CDCl}_{3}\right) \delta: 1.05(6 \mathrm{H}, \mathrm{d}, J=6.5$ $\mathrm{Hz}), 1.10-1.80(24 \mathrm{H}, \mathrm{m}), 2.03(2 \mathrm{H}, \mathrm{dq}, J=14.0,3.0 \mathrm{~Hz}), 2.28-2.50(4 \mathrm{H}, \mathrm{m}), 2.57-2.70(2 \mathrm{H}, \mathrm{m}), 2.87(2 \mathrm{H}, \mathrm{q}, J=$ $6.5 \mathrm{~Hz}), 4.78(2 \mathrm{H}, \mathrm{s}) ;{ }^{13} \mathrm{C}$ NMR $\delta: 18.9,25.0,25.4,26.3,29.1,29.4,34.9,37.3,53.6,55.6,70.4,172.9$. HRFABMS [M+H] $]^{+}$: Found, 423.3213, Calcd. for $\mathrm{C}_{24} \mathrm{H}_{43} \mathrm{~N}_{2} \mathrm{O}_{4}: 423.3224$.

\section{Supplementary data}

Supplementary data associated with this article can be found, in the online version, at http://dx.doi

\section{Acknowledgement}

This work was supported in part by a Grant-in-aid from the Japan Society for the Promotion of Science (24580160) to H. M.

\section{References and notes}

1. Strunz, G. M.; Findlay, J. A. The Alkaloids, Brossi, A., Eds; Academic Press: New York, 1985, Vol. 26, pp.89.

2. Koulocheri, S. D.; Pitsinos, E. N.; Haroutounian, S. A. Curr. Org. Chem. 2008, 12, 1454.

3. (a) Tadano, K.; Takao, K.; Nigawara, Y.; Nishio, E.; Takagi, I.; Maeda, K.; Ogawa, S. Synlett 1993, 565. (b)

Takao, K.; Nigawara, E.; Nishio, E.; Takagi, I.; Maeda, K.; Tadano, K.; Ogawa, S. Tetrahedron 1994, 50, 5681. 
4. Yokoyama, H.; Otaya, K.; Kobayashi H.; Miyazawa, M.; Yamaguchi, S.; Hirai, Y. Org. Lett. 2000, 2427.

5. Rall, G. J. H.; Smalberger, T. M.; de Waal, H. L. Tetrahedron Lett. 1967, 8, 3465.

6. Smalberger, T. M.; Rall, G. J. H.; de Waal, H. L.; Arndt, R. R. Tetrahedron 1968, 24, 6417.

7. Recent examples of synthesis of (+)-azimic acid (2): (a) Bates, R. W.; Kasinathan S. Tetrahedron 2013, 69, 3088. (b) Xiao, K. -J.; Liu, L. -X.; Huang, P. -Q. Tetrahedon: Asymmetry 2009, 20, 1181. (c) Leverett, C. A.; Cassidy, M. P.; Padwa, A. J. Org. Chem. 2006, 71, 8591. (d) Cassidy, M. P.; Padwa, A. Org. Lett. 2004, 6 4029.

8. Sato, T.; Aoyagi, S.; Kibayashi, C. Org. Lett. 2003, 5, 3839.

9. Makabe, H.; Looi, K. K.; Hirota, M. Org. Lett. 2003, 5, 27.

10. Grubbs, R. H.; Chang, S. Tetrahedron 1998, 54, 4413.

11. Masuda, Y.; Tashiro, T.; Mori. K. Tetrahedon: Asymmetry 2006, 17, 3380.

12. Shiina, I. Chem. Rev. 2007, 107, 239.

13. Inanaga, J.; Hirata, K.; Saeki, H.; Katsuki, T.; Yamaguchi, M. Bull. Chem. Soc. Jpn. 1979, 52, 1989. 


\section{Graphical Abstract}

\section{Total synthesis of $(+)$-azimine via diastereselective aminopalladation}

Yuji Korogome, Masaya Kogiso, Looi Kok Kong, Yasunao Hattori, Hiroyuki Konno, Mitsuru Hirota, and Hidefumi Makabe

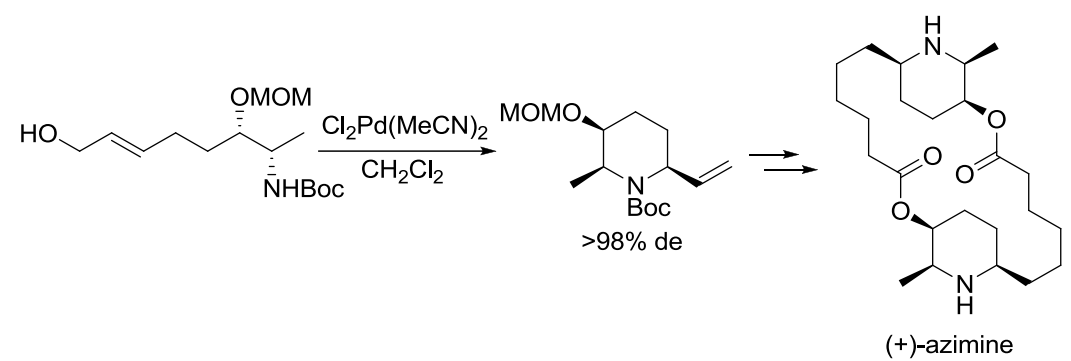

